\title{
A Survey of X-ray-Emitting IRAS Galaxies: A New Class of Objects or Hidden AGNs?
}

\section{Dennefeld}

Institut d'Astrophysique, 98 bis Bd. Arago, F-75014 Paris, France

\section{Introduction}

A cross-correlation between the IRAS PSC and the ROSAT All-Sky Survey has provided 242 sources detected in both surveys (Boller et al. 1992). About half of these have redshifts in the literature and a spectral classification. An unexpected result was the discovery of many galaxies with $\mathrm{X}$-ray luminosities up to a few $10^{43} \mathrm{ergs} \mathrm{s}^{-1}$, yet without Seyfert characteristics in their optical spectra (e.g., Boller et al. 1993). To investigate this problem further and evaluate the frequency of occurrence of these peculiar objects, a spectroscopic follow-up has been undertaken to:

- Determine velocities and luminosities for all the unclassified objects.

- Reevaluate the classification of high X-ray luminosity objects previously not recognized as Seyferts. In particular, spectral resolution or coverage and variability are important parameters to consider if one wants to detect faint signs of Seyfert activity.

\section{Observations and Classification}

Low-dispersion spectroscopy now has been obtained for 140 objects, with some of them observed several times. The northern objects were observed at HauteProvence Observatory, the southern ones at ESO La Silla. The spectral resolution was usually $11 \AA$, corresponding to a velocity resolution of about $250 \mathrm{~km} \mathrm{~s}^{-1}$ per pixel at $\mathrm{H} \alpha$. A higher spectral-resolution survey is also under way for the most interesting objects.

While some classical Sy 1 are easily recognized (although very few have permitted line widths larger than $1000 \mathrm{~km} \mathrm{~s}^{-1}$ ), a large fraction of objects have unresolved lines and are not directly recognizable as Seyferts. Use of line intensity ratios in standard diagnostic diagrams revealed only a few additional Seyfert galaxies.

A significant fraction of objects therefore do not show any signs of Seyfert activity at all in their optical spectra. Those are distributed over the whole $\mathrm{X}$-rays luminosity range, but are more numerous at lower luminosities.

Although the demarcation between AGN and 'normal' galaxies is not precisely defined in classical diagnostic diagrams, and many galaxies show complex spectra, a fair number of them can be considered as having secure classification. 


\section{Discussion}

The main features of the sample can be summarized as follows:

- Most of the spectra are heavily reddened, showing large Balmer decrements, red continua, and strong dust absorption features. In this respect, the spectra are similar to those from an $I R A S$-selected, non-X-ray emitting sample of galaxies (Dennefeld, in preparation).

- Conspicuous Sy 1 are rare, and a large proportion of Sy 1.8 or Sy 1.9 are found. A significant number of narrow-line Sy 1 galaxies are detected $(7 \%$ of total, and $30 \%$ of the Sy 1), a fraction much larger than in samples not directly selected from X-rays surveys.

- Excitation is generally low (few Sy 2), and the [O I]/H $\alpha$ ratio is low also, which excludes LINERs in most cases.

It is suggested that the peculiarities of this sample are due to the large amount of dust present in IRAS galaxies.

Two possibilities can be considered for the origin of the $\mathrm{X}$-rays emission for those galaxies which do not exhibit Sy features in their spectra.

1. No AGN is present in those objects which are powered by starbursts only. This is not contradictory with line widths of a few hundred $\mathrm{km} \mathrm{s}^{-1}$, as signs of interactions and mergers are well established in IRAS galaxies. Furthermore, Terlevich and collaborators have shown that both Fe II emission and moderate $[\mathrm{O}$ I] $/ \mathrm{H} \alpha$ ratios can be produced by starbursts. In this case, the $\mathrm{X}$-ray emission should have the same origin. How far in luminosity this is possible is presently being investigated.

2. An AGN is present, but is dominated by the starburst in the visible and/or hidden by the large amounts of dust, especially when it is in its low state. In this case, the observed soft X-rays (presumed to be due to the AGN) have to be scattered towards us. The intrinsic X-ray luminosity would then be an order of magnitude larger, and so would the AGNs optical component. In this picture, NLS1 properties could be related to the soft $\mathrm{X}$-ray component and to the presence of large amounts of dust. Variability might be the main reason for the non-detection of the AGN component. A specific observational effort to detect the presence of an AGN has already provided interesting cases of variability, either in X-rays or in the optical domain.

Whether only one or both of the suggested explanations are applicable, these highly obscured objects deserve a special effort to determine their contribution to the luminosity function of AGNs and to the soft X-ray background.

\section{References}

Boller, Th., et al. 1992, A\&A, 261, 57.

Boller, Th., et al. 1993, in 'First Light in the Universe', Ed. Frontieres, p. 339. 